\title{
Background galaxies as reddening probes throughout the Magellanic Clouds
}

\author{
C. M. Dutra ${ }^{1,3}$, E. Bica ${ }^{1,3}$, J. J. Clariáa ${ }^{2,3}$, A. E. Piatti ${ }^{2,3}$, and A. V. Ahumada ${ }^{2,3}$ \\ 1 Instituto de Fisica-UFRGS, CP 15051, CEP 91501-970 POA - RS, Brazil \\ 2 Observatorio Astronómico de Córdoba, Laprida 854, 5000, Córdoba, Argentina \\ 3 Visiting Astronomer, Complejo Astronómico El Leoncito operated under agreement between the Consejo \\ Nacional de Investigaciones Científicas y Técnicas de la República Argentina and the National Universities \\ of La Plata, Córdoba and San Juan
}

Received 3 January 2001 / Accepted 23 March 2001

\begin{abstract}
We study the spectral properties in the range $3600 \AA-6800 \AA$ of the nuclear region of galaxies behind the Magellanic Clouds. The radial velocities clarified the nature of the objects as background galaxies or extended objects belonging to the Clouds. For most galaxies behind the main bodies of the LMC and SMC, radial velocities were measured for the first time. In the present sample typical LMC background galaxies are nearby $\left(4000<V\left(\mathrm{~km} \mathrm{~s}^{-1}\right)<6000\right)$, while SMC's are considerably more distant $\left(10000<V\left(\mathrm{~km} \mathrm{~s}^{-1}\right)<20000\right)$. We determine the reddening in each line of sight by matching a reddening-free galaxy template with comparable stellar population. For the LMC main body we derive a combined Milky Way and internal reddening value $E(B-V)_{\mathrm{MW}+\mathrm{i}}=0.12 \pm 0.10$, while for the SMC $E(B-V)_{\mathrm{MW}+\mathrm{i}}=0.05 \pm 0.05$. By subtracting Milky Way reddening values for galaxies projected onto the surroundings of each Cloud, we estimate average internal reddening values $\Delta E(B-V)_{\mathrm{i}}=0.06$ and 0.04 , respectively for the main bodies of the LMC and SMC. The Clouds are optically thin, at least in the directions of the studied background galaxies which are often difficult to be identified as such on ESO/SERC sky survey images. Nevertheless, more reddened zones may occur where it is difficult to identify galaxies.
\end{abstract}

Key words. ISM: dust, extinction - Galaxy: general - galaxies: Magellanic Clouds, ISM, stellar content

\section{Introduction}

The galactic reddening distribution was initially modeled as a function of galactic latitude in terms of a Cosecant Law (Sandage 1973 and references therein). A dependence on galactic longitude was derived by de Vaucouleurs et al. (1976). The polar cap zero points have been a matter of debate with estimates ranging from reddening free to $E(B-V)=0.05$. These models do not take into account local reddening variations, since the dust distribution can be very patchy, including high galactic latitudes where many discrete clouds occur (e.g. Reach et al. 1998). The empirical formulation by Burstein \& Heiles (1978, 1982) improved the reddening distribution description by relating HI column density and galaxy counts to reddening. Nevertheless the H I and dust contents do not scale in the same way everywhere, in particular in cold dense clouds where hydrogen becomes mostly molecular.

Send offprint requests to: C. M. Dutra,

e-mail: dutra@if.ufrgs.br
Recently, Schlegel et al. (1998, hereafter SFD98) provided a new estimator of galactic reddening by means of a full-sky $100 \mu \mathrm{m}$ IRAS/ISSA map which was converted to dust column density by using a dust colour temperature map ( $17 \mathrm{~K}$ to $21 \mathrm{~K}$ ) derived from 100 and $240 \mu \mathrm{m}$ COBE/DIRBE maps. The dust emission map is calibrated in terms of $E(B-V)$ reddening values using determinations from early type galaxies by means of the $(B-V)$ vs. Mg2 relation. SFD98's dust emission reddening $E(B-V)_{\mathrm{FIR}}$ appears to be sensitive to the dust content of the cold dense clouds and their accumulation in different lines of sight. Dutra \& Bica (2000) compared $E(B-V)_{\text {FIR }}$ values with the reddening $E(B-V)$ measured from the stellar content of globular and old open clusters in the Galaxy. It was concluded that differences between these reddening values most probably arise from dust distribution in the cluster foreground and background.

The stellar content of background galaxies offers an opportunity to analyse the total Milky Way dust column in a given line of sight, as well as those of the Magellanic 
Clouds. Recently the NED database included a facility tool to determine SFD98's $E(B-V)_{\text {FIR }}$ in any direction. However for the main bodies of the LMC and SMC NED adopted uniform foreground reddening values of $E(B-$ $V)_{\mathrm{FIR}}=0.075$ and $E(B-V)_{\mathrm{FIR}}=0.037$, respectively. These values are from the average dust emission in their surroundings (SFD98). We emphasize that in the present study we use SFD98's original facility tool (dust-getval.f) and the whole sky dust emission reddening maps, which include the internal dust emission structure of the LMC

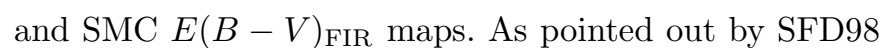
they have not analysed the $E(B-V)_{\mathrm{FIR}}$ values in the Clouds.

The Magellanic Clouds cover a significant portion of the sky and their background galaxies have not yet been studied in detail due in part to reddening and crowding effects. Oestreicher et al. (1995) mapped the galactic reddening in the direction of the LMC by means of $U B V$ photometry of foreground galactic stars. They obtained a mean reddening of $E(B-V)_{\mathrm{MW}}=0.06 \pm 0.02$. For the $\mathrm{SMC}$ the mean foreground reddening is $E(B-V)_{\mathrm{MW}} \approx$ 0.03 considering colour-magnitude diagrams of clusters in the outer parts of the SMC such as K3, L1 and NGC 121 (Westerlund 1990). Oestreicher \& Schmidt-Kaler (1996) studied the internal reddening distribution of the LMC with dust clouds in the range $0.04<E(B-V)_{\mathrm{i}}<$ 0.40. Reddening estimates using background galaxies by means of count methods have been applied for the SMC (Wesselink 1961; Hodge 1974; MacGillivray 1975) and LMC (Gurwell \& Hodge 1990). These studies provide very high reddening values in some regions which seem to arise from count losses owing to star crowded fields and extended objects belonging to the Clouds.

In the present study, we observe and analyse integrated spectra of nuclear regions of galaxies behind the main bodies of the Clouds to probe reddening in those lines of sight. We take into account different stellar populations by using template spectra. For comparison purposes we also observed galaxy spectra in the surroundings of the Clouds and towards the Galactic South Polar Cap. In Sect. 2 we present the samples in the directions of the Clouds and South Polar Cap. In Sect. 3 we describe the observations and reductions. In Sect. 4 we establish the nature of the objects. Most of them turn out to be galaxies, but some are extended objects belonging to the Clouds. In Sect. 5 we compare the present galaxy spectra with those of stellar population templates from Bica (1988, hereafter B88), and provide some new templates as well. In Sect. 6 we derive the reddening value for each galaxy behind the Magellanic Clouds and discuss their distribution and overall tranparency of the Clouds. Concluding remarks are given in Sect. 7.

\section{The samples}

In the main bodies of the Magellanic Clouds it is often difficult to establish the morphological type of a galaxy using ESO/SERC Schmidt plates and Digitized Sky Survey at- lases. In some cases is not possible to distinguish a galaxy from an extended object belonging to the Clouds, such as a compact H II region or star cluster.

\subsection{Objects towards the Magellanic Clouds}

We selected galaxies behind the Magellanic Clouds and also included objects with uncertain classification and/or excluded from the revised and extended catalogues of extended objects in the SMC (Bica \& Schmitt 1995; Bica \& Dutra 2000) and LMC (Bica et al. 1999) to establish their nature. These LMC or SMC main body objects are: (i) SMC-DEM92(AM0054-744ne), LMCDEM225 and LMC-DEM329 from the SMC and LMC catalogues of emission nebulae by Davies et al. (1976); (ii) HS 75-8, HS 75-10, HS 75-13, HS 75-18, HS 75-20, HS 75-22, HS 75-23 and HS 75-25 from the catalogue of galaxies behind the SMC by Hodge \& Snow (1975); (iii) HS 17, HS 45, HS 257, HS 356, HS 394, HS 449 and HS 451 from the LMC star cluster catalogue by Hodge \& Sexton (1966); (iv) SL887 from the LMC star cluster catalogue by Shapley \& Lindsay (1963); (v) OHSC3 from the LMC star cluster catalogue by Olszewski et al. (1988); and (vi) HW 60 from the SMC star cluster catalogue by Hodge \& Wright (1974).

Figures 1 and 2 show the angular distribution of the observed objects towards the SMC and LMC respectively. We distinguish the literature galaxies that we observed (LEDA and/or NED extragalactic databases) from the objects whose nature will be established in the present study. The latter objects are excellent reddening probes for the central regions of the Clouds. A preliminary discussion of part of the Magellanic Clouds sample was given in Dutra et al. (1998).

The 43 selected objects are presented in Table 1 , by columns: (1) designation, (2) and (3) J2000 equatorial coordinates, (4) and (5) galactic coordinates, (6) total magnitude $B_{\mathrm{T}}$ (LEDA/NED), (7) exposure time, (8) radial velocity measurement, (9) LEDA and/or NED radial velocity, and (10) LEDA/NED morphological type or estimated by ourselves on ESO/SERC plates when possible. Additional objects included in Table 1 are discussed subsequently.

\subsection{South Polar Cap}

The South Polar Cap sample $\left(b<-70^{\circ}\right)$ consists of 13 early type galaxies to minimize stellar population variations. SFD98 pointed out the existence of low reddening regions between cirrus filaments near the Galactic Poles, and in some regions outside the Polar Caps as well at intermediate latitudes. Some of the latter regions have $E(B-V)_{\mathrm{FIR}}$ values four times less than those estimated for the Polar Caps averaged over regions of diameter $10^{\circ}$, which are $E(B-V)_{\mathrm{FIR}}=0.015$ and 0.018 , respectively for the Northern and Southern Polar Caps. We observed these galaxies to create reddening-free galaxy templates 
Table 1. The observed objects towards the Magellanic Clouds

\begin{tabular}{|c|c|c|c|c|c|c|c|c|c|}
\hline Object & $\begin{array}{c}\text { RA (2000) } \\
\text { h:m:s }\end{array}$ & $\begin{array}{c}\text { Dec }(2000) \\
\circ:^{\prime}:^{\prime \prime} \\
\end{array}$ & $\begin{array}{c}\ell \\
\left({ }^{\circ}\right) \\
\end{array}$ & $\begin{array}{c}b \\
\left({ }^{\circ}\right) \\
\end{array}$ & $B_{\mathrm{t}}$ & $\begin{array}{l}\text { Exp } \\
\text { (s) }\end{array}$ & $\begin{array}{c}V \\
\left(\mathrm{~km} \mathrm{~s}^{-1}\right) \\
\end{array}$ & $\begin{array}{c}V_{\text {lit }} \\
\left(\mathrm{km} \mathrm{s}^{-1}\right)\end{array}$ & Type \\
\hline & & & & & main 1 & & & & \\
\hline HS $75-8$ & $00: 51: 08$ & $-73: 39: 22$ & 302.96 & -43.47 & & $4 \times 900$ & 19650 & - & $\mathrm{E}$ \\
\hline AM0054-744sw & $00: 55: 49$ & $-74: 30: 58$ & 302.53 & -42.61 & & $2 \times 900$ & 10685 & - & $\mathrm{E}$ \\
\hline SMC-DEM92,AM0054-744ne & 00:56:01 & $-74: 30: 40$ & 302.52 & -42.61 & & $3 \times 900$ & 10369 & - & $\mathrm{E} / \mathrm{SO}$ \\
\hline HS $75-20$ & $00: 59: 10$ & $-74.02: 39$ & 302.20 & -43.07 & & $5 \times 900$ & 19017 & - & $\mathrm{E}$ \\
\hline HS $75-22$ & 01:06:03 & $-73: 59: 52$ & 301.55 & -43.09 & & $2 \times 900$ & 9890 & - & $\mathrm{E}$ \\
\hline HS $75-23$ & $01: 06: 07$ & $-74: 07: 42$ & 301.56 & -42.96 & & $5 \times 900$ & 18950 & - & $\mathrm{E}$ \\
\hline HW 60 & $01: 09: 27$ & $-72: 22: 21$ & 301.01 & -44.69 & & $3 \times 900$ & 17665 & - & $\mathrm{S}$ \\
\hline HS 75-25,PMN J0111-7302 & $01: 11: 33$ & $-73: 02: 12$ & 300.89 & -44.01 & & $3 \times 900+600$ & 19736 & - & $\mathrm{E}$ \\
\hline \multirow[t]{2}{*}{ NGC 643B,ESO 29G53,IRAS 01384-7515 } & 01:39:14 & $-75: 00: 41$ & 298.82 & -41.73 & & $2 \times 900$ & 4006 & 3966 & \\
\hline & \multicolumn{8}{|c|}{ SMC surroundings } & \\
\hline ESO 28G12,IRAS 00160-7325 & $00: 18: 20$ & $-73: 09: 08$ & 306.24 & -43.76 & 14.90 & $2 \times 600$ & 6200 & 6326 & S0-a \\
\hline HS $75-10$ & $00: 52: 34$ & $-70: 28: 17$ & 302.79 & -46.66 & & $3 \times 900$ & 18360 & - & $\mathrm{E}$ \\
\hline NGC 406 ,ESO 51G18,IRAS 01057-7008 & $01: 07: 24$ & $-69: 52: 35$ & 300.91 & -47.19 & 13.02 & $3 \times 600$ & 1391 & 1508 & $\mathrm{Sc}$ \\
\hline ESO 52IG1-NED1 & $01: 24: 49$ & $-68: 37: 21$ & 298.37 & -48.21 & 14.76 & $2 \times 900$ & 10778 & 11100 & SO-a \\
\hline NGC 802 , ESO 52 G13 & 01:59:06 & $-67: 52: 16$ & 293.50 & -48.00 & 14.09 & $2 \times 900$ & 1723 & 1504 & $\mathrm{SO}-\mathrm{a}$ \\
\hline NGC 813 , ESO 52 G16 & 02:01:37 & $-68: 26: 21$ & 293.52 & -47.38 & 13.78 & $600+420$ & 8188 & 8160 & SO-a \\
\hline \multirow[t]{2}{*}{ IC 5339, ESO 77G26,Fairall1051 } & 23:38:05 & $-68: 26: 35$ & 312.74 & -47.26 & 14.46 & $2 \times 900$ & 12250 & 12328 & E-SO \\
\hline & \multicolumn{8}{|c|}{ SMC extended objects } & \\
\hline 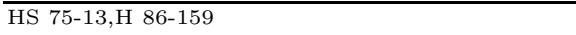 & $00: 55: 12$ & $-72: 40: 57$ & 302.54 & -44.44 & & $3 \times 900$ & 180 & - & Star cluster \\
\hline \multirow[t]{2}{*}{ SMC-N63,HS 75-18 } & $00: 58: 17$ & $-72: 38: 50$ & 302.21 & -44.47 & & $2 \times 900$ & 132 & - & H II Region \\
\hline & \multicolumn{8}{|c|}{ LMC main body } & \\
\hline ESO 55G33 & $04: 38: 51$ & $-69: 30: 25$ & 281.49 & -36.69 & 14.39 & $2 \times 900$ & 5470 & - & So \\
\hline NGC 1669, ESO 84G38 & 04:43:00 & $-65: 48: 53$ & 276.96 & -37.57 & 14.78 & $900+2 \times 600$ & 5580 & - & $\mathrm{Sa}$ \\
\hline NGC 1809, ESO 56 G48 & 05:02:05 & $-69: 34: 04$ & 280.76 & -34.75 & 13.19 & $3 \times 900$ & 1233 & 1301 & Sc \\
\hline ESO 33G11 & $05: 05: 07$ & $-73: 39: 08$ & 285.39 & -33.34 & 14.35 & $2 \times 900$ & 4550 & - & SBaR \\
\hline NEW GALAXY 1 & $05: 07: 38$ & $-68: 23: 03$ & 279.21 & -34.57 & & $2 \times 900+420$ & 5782 & - & \\
\hline HS 257 ,GSC 916600034 & $05: 22: 45$ & $-70: 10: 29$ & 280.97 & -32.88 & & $2 \times 900$ & 5560 & - & \\
\hline LMC-DEM225,IRAS 05319-6723 & $05: 31: 49$ & $-67: 21: 32$ & 277.51 & -32.51 & & $3 \times 600$ & 1376 & - & \\
\hline IRAS 05338-6645 & $05: 33: 52$ & $-66: 43: 18$ & 276.74 & -32.39 & & $3 \times 900$ & 4320 & - & \\
\hline HS 356,ESO 56G154,KMHK1096,RXJ 0534.0-7145 & $05: 33: 58$ & $-71: 45: 20$ & 282.62 & -31.70 & & $900+600$ & 7150 & 7255 & \\
\hline HS 394 & 05:42:02 & $-70: 54: 15$ & 281.52 & -31.18 & & $3 \times 900$ & 4567 & - & \\
\hline \multirow[t]{2}{*}{$\underline{\text { LMC-DEM329,IRAS 05522-6952 }}$} & 05:51:42 & $-69: 55: 51$ & 280.31 & -30.46 & & $2 \times 900$ & 4380 & - & \\
\hline & & & & & surrour & & & & \\
\hline HS 17,RXSJ 043612.5-682236,HP 99-653 & $04: 36: 15$ & $-68: 22: 10$ & 280.27 & -37.33 & & $4 \times 900$ & 19250 & - & \\
\hline HS 45,IRASF 04521-7333 & 04:51:09 & $-73: 28: 42$ & 285.59 & -34.33 & & $3 \times 900$ & 7565 & - & \\
\hline $\mathrm{ESO} 33 \mathrm{G} 2$ & $04: 55: 59$ & $-75: 32: 28$ & 286.77 & -33.29 & 14.66 & $2 \times 900$ & 5408 & 5467 & So \\
\hline ESO 33G3 & $04: 57: 47$ & $-73: 13: 51$ & 285.11 & -33.97 & 14.25 & $2 \times 600$ & 7664 & 7677 & $\mathrm{E}$ \\
\hline NGC 1765, ESO 119 G24 & $04: 58: 24$ & $-62: 01: 41$ & 271.83 & -36.89 & 13.97 & $2 \times 900$ & 8846 & 8758 & $\mathrm{E}$ \\
\hline ESO $15 \mathrm{G} 18$ & 05:04:58 & $-81: 18: 38$ & 293.89 & -30.73 & 14.29 & $2 \times 900$ & 4926 & 4903 & $\mathrm{E}$ \\
\hline ESO $119 \mathrm{G} 48$ & $05: 14: 36$ & $-61: 28: 54$ & 270.78 & -35.09 & 13.51 & $2 \times 900$ & 4549 & 4548 & SO-a \\
\hline HS 449 , GH 90-060055-6840 & $06: 00: 43$ & $-68: 40: 09$ & 278.81 & -29.72 & & $4 \times 900$ & 11305 & - & \\
\hline NGC 2187A,ESO 57G68sw,AM 0604-693sw & $06: 03: 44$ & $-69: 35: 18$ & 279.86 & -29.44 & 12.94 & $2 \times 900$ & 3769 & 3963 & $\mathrm{Sa}$ \\
\hline NGC 2187B,ESO 57G68ne,AM 0604-693ne & $06: 03: 52$ & $-69: 34: 41$ & 279.85 & -29.43 & 13.16 & $2 \times 900$ & 4519 & 4470 & $\mathrm{E}$ \\
\hline HS 451 & $06: 05: 28$ & $-67: 07: 10$ & 277.03 & -29.28 & & $3 \times 900$ & 7931 & - & \\
\hline SL 887 & $06: 21: 01$ & $-72: 35: 34$ & 283.30 & -28.06 & & $3 \times 900+600$ & 11466 & - & \\
\hline ESO 58G19 & $06: 52: 57$ & $-71: 45: 44$ & 282.64 & -25.55 & 13.45 & $2 \times 900$ & 4271 & 4251 & SO-a \\
\hline & & & & & xtendec & & & & \\
\hline OHSC3,KMHK362 & $04: 56: 36$ & $-75: 14: 29$ & 287.42 & -33.36 & & $4 \times 900$ & 158 & - & Star cluster \\
\hline & & & & & parison & & & & \\
\hline NGC 1381 & $03: 36: 31$ & $-35: 17: 39$ & 236.467 & -54.039 & 12.71 & $2 \times 600$ & 1676 & 1776 & So \\
\hline NGC 1399 & $03: 38: 29$ & $-35: 26: 58$ & 236.714 & -53.636 & 10.33 & $2 \times 900$ & 1424 & 1434 & $\mathrm{E}$ \\
\hline NGC 1411 & $03: 38: 45$ & $-44: 06: 00$ & 251.02 & -52.52 & 12.18 & $2 \times 900$ & 1100 & 1022 & E-So \\
\hline NGC 1404 & $03: 38: 52$ & $-35: 35: 35$ & 236.953 & -53.555 & 10.89 & $2 \times 600$ & 1970 & 1926 & $\mathrm{E}$ \\
\hline NGC 1427 & $03: 42: 19$ & $-35: 23: 37$ & 236.598 & -52.854 & 11.84 & $2 \times 600$ & 1328 & 1425 & $\mathrm{E}$ \\
\hline NGC 1600 & $04: 31: 40$ & $-05: 05: 10$ & 200.416 & -33.242 & 12.04 & $2 \times 600$ & 4703 & 4737 & $\mathrm{E}$ \\
\hline NGC 6758 & $19: 13: 52$ & $-56: 18: 33$ & 340.573 & -25.318 & 12.58 & $2 \times 600$ & 3489 & 3408 & $\mathrm{E}$ \\
\hline IC 4889 & $19: 45: 16$ & $-54: 20: 37$ & 343.538 & -29.42 & 12.02 & $2 \times 600$ & 2490 & 2521 & $\mathrm{E}$ \\
\hline IC 1459 & 22:57:09 & $-36: 27: 37$ & 4.665 & -64.106 & 11.17 & $2 \times 600$ & 1726 & 1679 & $\mathrm{E}$ \\
\hline & & & & & h Polar & & & & \\
\hline NGC 148 & $00: 34: 16$ & $-31: 47: 10$ & 340.648 & -84.029 & 13.24 & $3 \times 600$ & 1705 & 1516 & S0 \\
\hline NGC 155 & $00: 34: 40$ & $-10: 45: 59$ & 108.57 & -73.169 & 14.28 & $5 \times 900$ & 6101 & 6173 & So \\
\hline NGC 163 & 00:36:00 & $-10: 07: 17$ & 110.121 & -72.608 & 13.92 & $2 \times 900$ & 5892 & 5981 & $\mathrm{E}$ \\
\hline NGC 179 & $00: 37: 46$ & $-17: 50: 56$ & 103.462 & -80.2 & 14.29 & $2 \times 900$ & 6192 & 6006 & E-SO \\
\hline NGC 277 & $00: 51: 17$ & $-08: 35: 48$ & 122.814 & -71.468 & 14.73 & $900+600$ & 4120 & 4327 & E-SO \\
\hline IC 1633 & 01:09:55 & $-45: 55: 52$ & 293.099 & -70.843 & 12.55 & $2 \times 600$ & 7437 & 7242 & $\mathrm{E}$ \\
\hline $\mathrm{ESO} 476 \mathrm{G} 4$ & $01: 21: 07$ & $-26: 43: 36$ & 211.145 & -83.373 & 13.86 & $2 \times 900$ & 5922 & 5839 & E-SOB \\
\hline ESO $352 \mathrm{G} 55$ & $01: 21: 33$ & $-33: 09: 23$ & 257.572 & -81.133 & 14.63 & $2 \times 900$ & 3747 & 3539 & E-SO \\
\hline ESO $542 \mathrm{G} 15$ & $01: 27: 14$ & $-21: 46: 24$ & 181.527 & -80.258 & 14.70 & $2 \times 900$ & 5532 & 5567 & SORing \\
\hline NGC 641 & $01: 38: 39$ & $-42: 31: 40$ & 273.994 & -71.847 & 13.42 & $2 \times 900$ & 6306 & 6454 & E-SO \\
\hline NGC 720 & 01:53:00 & $-13: 44: 20$ & 173.019 & -70.358 & 11.40 & $2 \times 600$ & 1563 & 1736 & $\mathrm{E}$ \\
\hline NGC 7736 & $23: 42: 26$ & $-19: 27: 09$ & 55.15 & -72.418 & 13.80 & $4 \times 900$ & 4492 & 4511 & So \\
\hline NGC 7761 & $23: 51: 29$ & $-13: 22: 53$ & 74.423 & -70.37 & 14.14 & $3 \times 900$ & 7087 & 7082 & $\mathrm{SO}$ \\
\hline
\end{tabular}




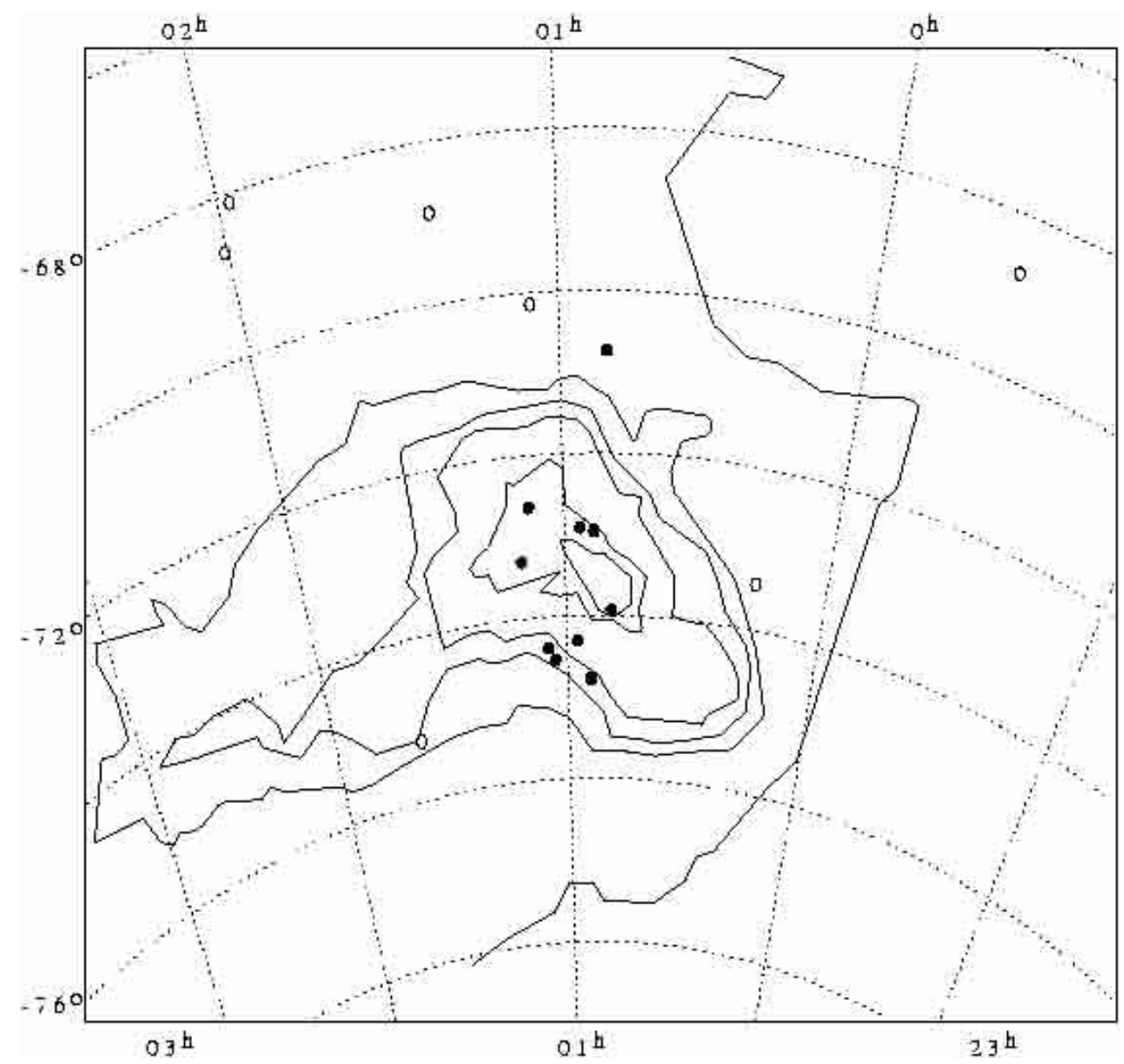

Fig. 1. Angular distribution of the observed SMC background known galaxies (open circles) and candidate galaxies (filled circles). The solid lines represent H I contours of 5, 50, 100, 150, 400 and 600 in units $10^{19}$ atoms cm $^{-2}$ from Mathewson \& Ford $(1984)$

under the same observational conditions as the Magellanic Clouds sample. We also observed 9 intermediate galactic latitude galaxies $\left(-64^{\circ}<b<-29^{\circ}\right)$ which are in common with those in B88's red stellar population templates in early type galaxies. The latter galaxies were observed for comparison purposes. The samples are given in Table 1.

\section{Observations and reductions}

The spectra were collected with the 2.15-meter telescope at the Complejo Astronómico El Leoncito (CASLEO, San Juan, Argentina) in December 1995 and October 1998. We employed a CCD camera attached to the REOSC spectrograph. The detector was a Tektronics chip of $1024 \times 1024$ pixels of size $24 \mu \times 24 \mu$. We used a grating of 300 grooves $\mathrm{mm}^{-1}$ producing an average dispersion of $\approx 143 \AA / \mathrm{mm}$ or $3.43 \AA /$ pixel. The spectral coverage was $3600 \AA-6800 \AA$. At least two exposures of each object were taken in order to correct for cosmic rays. The exposure times are given in Table 1. The standard stars EG 21 and LTT 3864 (Baldwin \& Stone 1984) were observed for flux calibrations. He-Ar lamp exposures were taken following that of the object or standard star for wavelength calibrations. The slit width was $4^{\prime \prime}$ providing a resolution $(F W H M)$ of $\approx 14 \AA$ from comparison lines. The slit was set in the E-W direction, and its length projected on the chip $\left(4.7^{\prime}\right)$ provided a wide range of pixel rows for background subtractions.

The reductions were carried out at the Instituto de Física, UFRGS (Porto Alegre-Brazil) and Observatorio Astronómico, Universidad Nacional de Córdoba (Córdoba-Argentina), with the IRAF package following standard procedures. The galaxy spectra were extracted along the slit according to the dimensions of each galaxy nuclear region. Typical extractions were $\approx 8-10^{\prime \prime}$.

Since the spectral resolution was chosen for stellar population purposes, it is not ideal for velocity measurements. At any rate, we measured velocities and the agreement with values in common with the literature is good (Table 1). In one case, ESO 28G12, the current LEDA and NED velocities are significantly different, and the present observation confirms the latter value (Table 1 ). The measured velocities were used to bring the galaxy spectra to the rest frame, which is necessary for the subsequent stellar population analysis.

Table 1 shows that the galaxies behind the SMC main body are mostly in the range $10000<V\left(\mathrm{kms}^{-1}\right)<$ 20 000, while those behind the LMC main body are mostly 


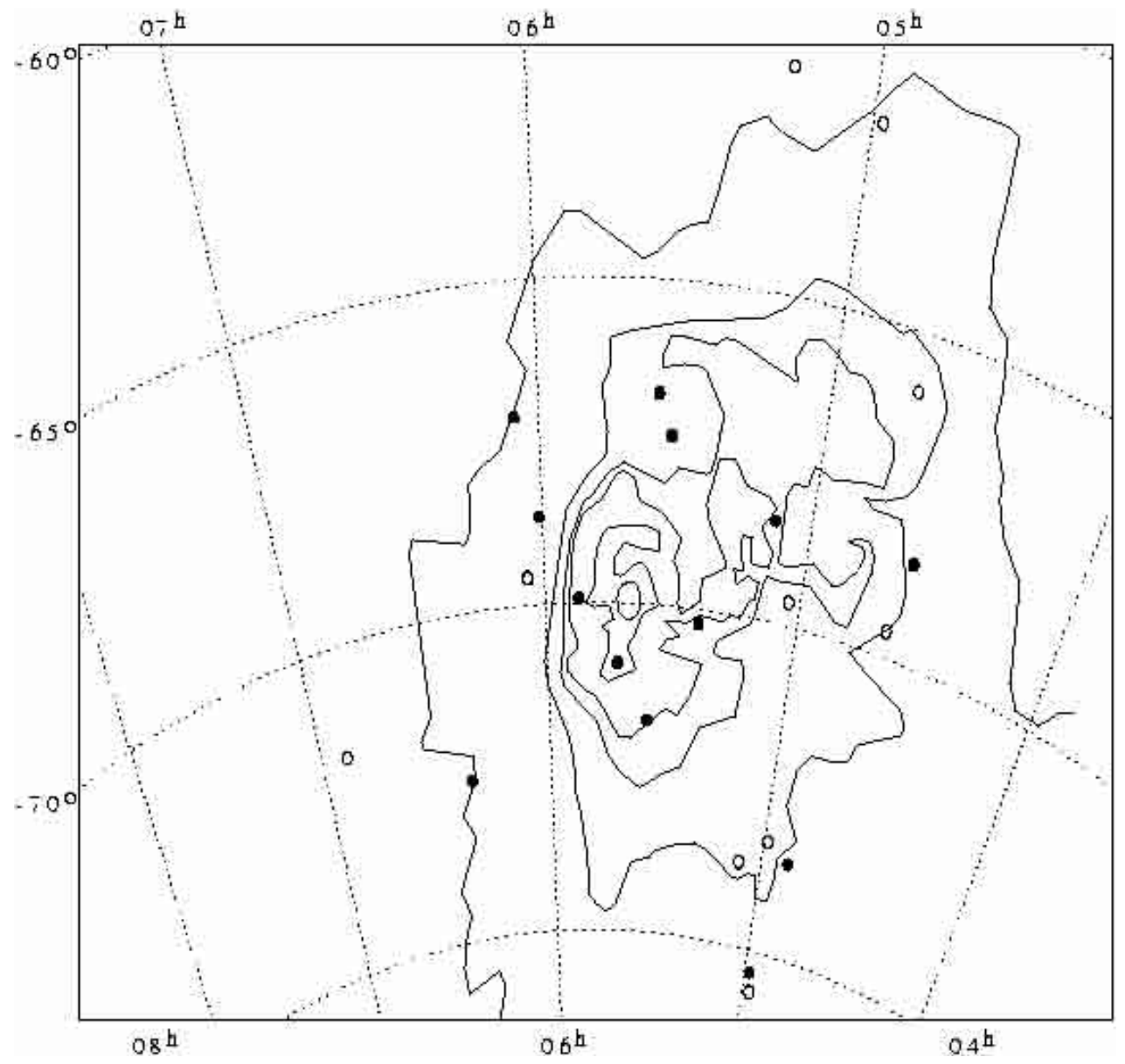

Fig. 2. Angular distribution of the observed LMC background known galaxies (open circles) and candidate galaxies (filled circles). The solid lines represent H I contours of 5, 50, 100, 150, 400 and 600 in units $10^{19}$ atoms cm $^{-2}$ from Mathewson \& Ford (1984)

in the range $4000<V\left(\mathrm{~km} \mathrm{~s}^{-1}\right)<6000$. Since we have not a priori selected galaxies differently between the two Clouds the result suggests a real effect, in the sense that towards the LMC a closer galaxy ensemble occurs, which has no counterpart in the SMC background.

Figures 3 and 4 show the resulting rest-frame flux calibrated spectra for the Clouds background galaxies, for which the present study established or confirmed their nature (Sect. 4). Most of these galaxies have red stellar population nuclei, but some are bluer and present features denoting recent star formation such as Balmer absorption lines (e.g. HS 75-20 in the SMC, Fig. 3) or emission lines typical of nuclear H II Regions (e.g. IRAS 05538-6645 in the LMC, Fig. 4).

\section{Nature of the objects towards the Clouds}

We established the nature of the objects towards the Clouds from the radial velocity measurements (Table 1). In the present study velocities resulted either $V>$ $1000 \mathrm{~km} \mathrm{~s}^{-1}$ or $V<200 \mathrm{~km} \mathrm{~s}^{-1}$, undoubtedly characterizing background galaxies and Magellanic Clouds' internal objects, respectively. Most of the objects observed from Hodge \& Snow's (1975) list are confirmed as galax- ies, except HS 75-13 and HS 75-18 (upper panel of Fig. 5) which turned out to be a SMC star cluster (H86-159) and a SMC H II region (SMC-N63), respectively (see Sect. 4.1). OHSC3 is confirmed as a LMC star cluster (upper panel of Fig. 5). The objects SMC-DEM92 and HW 60 in the SMC, and LMC-DEM225, LMC-DEM329, HS 17, HS 45, HS 257, HS 356, HS 394, HS 449, HS 451 and SL 887 in the LMC (originally classified as star clusters or H II regions) are shown to be galaxies. In the following we comment on the properties of some objects:

One observed galaxy was not catalogued previously (New Galaxy 1, in Table 1).

HW 60 appears to have a companion galaxy (New Galaxy 2, located at $\mathrm{J} 200001^{\mathrm{h}} 09^{\mathrm{m}} 23^{\mathrm{s}}-72^{\circ} 22^{\prime} 15^{\prime \prime}$ ) at $\approx 0.4^{\prime}$ to the northwest with dimensions $0.2^{\prime} \times 0.15^{\prime}$. They possibly form an interacting system, which is supported by the fact that the HW 60's spectrum is blue and presents strong emission lines, suggesting recent star formation (Fig. 3). Owing to the high radial velocity we do not have the $\mathrm{H}_{\alpha},[\mathrm{NII}]$ and $[\mathrm{SII}]$ region. Therefore, some nuclear activity cannot be ruled out.

SMC-DEM92 is the brighter member of the interacting pair AM 0054-744 (Arp \& Madore 1987). We also observed the companion AM 0054-744sw (Table 1, Fig. 3). 
Their radial velocities (Table 1) are comparable, supporting an interaction. A similar case is the interacting pair NGC 2187A and NGC 2187B (Table 1), which is also an entry in Arp \& Madore's catalogue.

In the SMC background the galaxy HS 75-25 is an Xray emitter (Haberl et al. 2000), see present Table 1 for coordinates and other designation. Behind the LMC are X-ray emitters corresponding to HS 17, HS 257 (Haberl \& Pietsch 1999) and HS 356 (Crampton et al. 1997). In particular, HS 356 in a rich LMC field has been catalogued not only as star cluster (Hodge \& Sexton 1966; Kontizas et al. 1990) but as a galaxy as well (Lauberts 1982).

ESO 52IG1 (Lauberts 1982) refers to a compact group of galaxies, studied by Sérsic (1974) - Se10/2, and Arp \& Madore (1987) - AM 0123-685. The present galaxy is the brightest member of the group (accurate coordinates in Table 1). We point out that the literature coordinates often refer to the group centre. The NED database currently lists the 3 brighter members, but all coordinates are systematically shifted $\approx 0.7^{\prime}$ to the northeast.

Some galaxies behind the Clouds are IRAS sources (Table 1).

\subsection{Discussion of the $L M C$ and $S M C$ extended objects}

Bica \& Schmitt (1995) and Bica \& Dutra (2000) indicated that the object SMC-N63 (Henize 1956) is also present in several other emission object catalogues as L61-331 (Lindsay 1961), SMC-DEM94 (Davies et al. 1976) and MA 1065 (Meyssonnier \& Azzopardi 1993). The present cross-identification of this object with HS 75-18 together with its CCD spectrum (upper panel of Fig. 5) conclusively establishes its nature as an H II region. The stellar content of the H II region was catalogued as the star cluster SMC-OGLE113 (Pietrzyński et al. 1998). The gas reddening including the foreground reddening can be derived from the emission line spectrum (upper panel of Fig. 5) using the Balmer decrement $F_{\mathrm{H}_{\alpha}} / F_{\mathrm{H}_{\beta}}=3.31$. Assuming case $\mathrm{B}$ of the recombination-line theory the intrinsic ratio is $\left(F_{\mathrm{H}_{\alpha}} / F_{\mathrm{H}_{\beta}}\right)_{0}=2.87$ (Osterbrock 1989), wherefrom we derive $E(B-V)=0.13$. This value is lower than SFD98's dust emission reddening estimate $E(B-V)_{\mathrm{FIR}}=0.37$. A possible explanation for this difference is that the molecular cloud related to this recent star forming region is located behind SMC-N63.

Integrated spectra of star clusters as compared to template cluster spectra of different ages and metallicities can provide parameters such as age and reddening (e.g. Piatti et al. 1998; Ahumada et al. 2000). In the present study the signal-to-noise ratios of the $\mathrm{H}$ 86-159 and OHSC3 spectra are not ideal for a detailed study of the cluster spectral features, but the spectral distribution can be compared to templates (lower panel of Fig. 5).

Using a deep plate from the CTIO 4-meter telescope Hodge (1986) catalogued the star cluster H 86159, and CCD images confirmed that (SMC-OGLE102 Pietrzyński et al. 1998). The present study cross-identifies
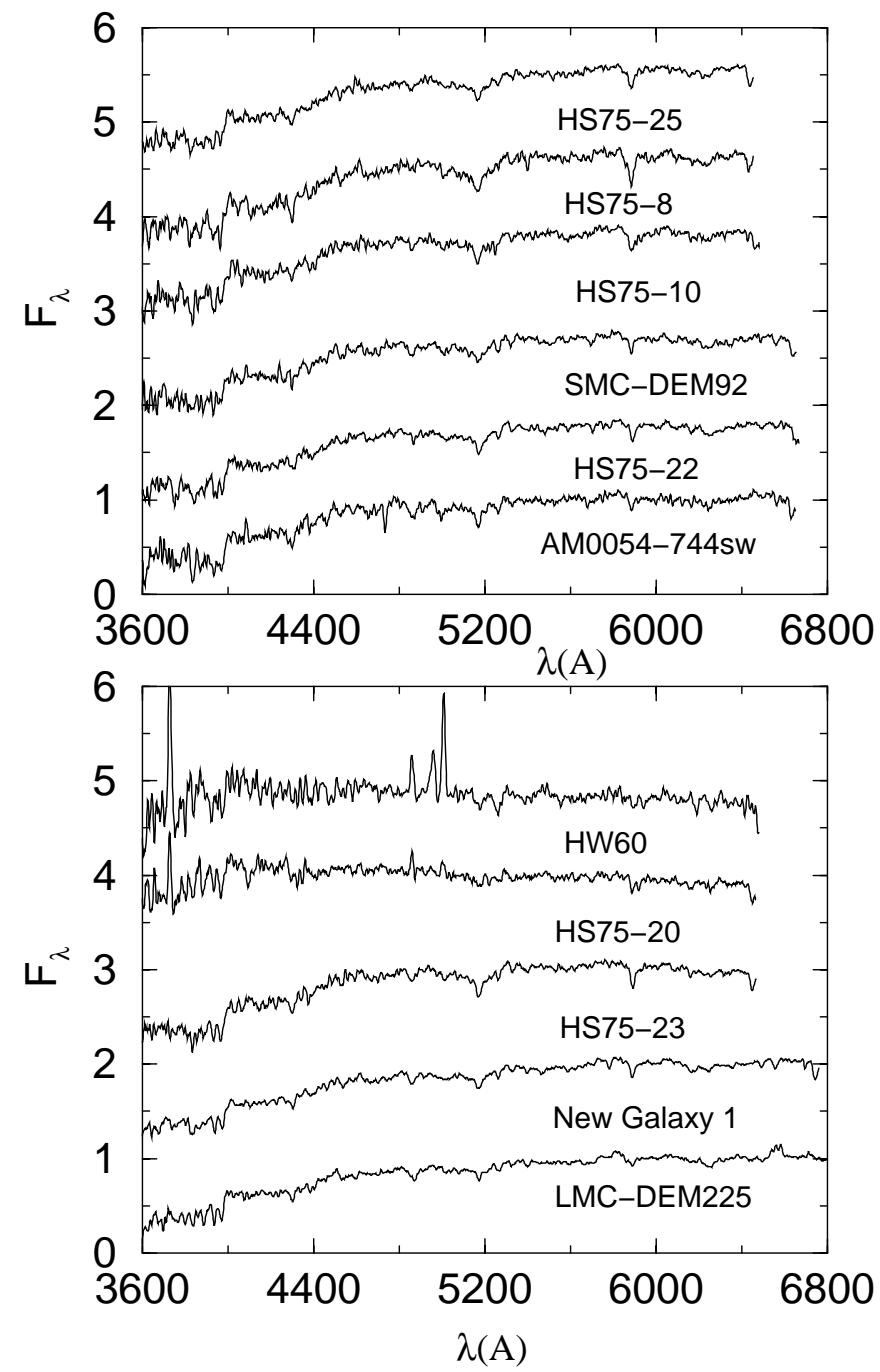

Fig. 3. Rest-frame spectra of objects which turned out to be or were confirmed as SMC background galaxies, together with two LMC's (New Galaxy 1 and LMC-DEM225)

the latter object with HS 75-13 and its spectrum (upper panel Fig. 5) confirms that one is not dealing with a galaxy. Recently de Oliveira et al. (2000) estimated a total reddening $E(B-V)_{\mathrm{MW}+\mathrm{SMC}}=0.10$ and age $\approx 500 \mathrm{Myr}$ for $\mathrm{H}$ 86-159 from a colour-magnitude diagram (CMD) extracted from the OGLE photometric database (Udalski et al. 1998). As comparison (lower panel of Fig. 5) we show the reddening-free LMC cluster templates LMC-Int1 and LMC-Int2, respectively of ages 1 and 2 Gyr (Dutra et al. 1999). By applying reddening corrections to the spectrum of H 86-159 following Seaton's (1979) extinction law, we derive $E(B-V)_{\mathrm{MW}+\mathrm{SMC}}=0.15$ and an age of 2 Gyr. In the cluster direction SFD98's dust emission reddening is $E(B-V)_{\mathrm{FIR}}=0.38$. The object is central in the SMC and star forming regions are nearby (traces of diffuse gas are denoted by [OII] $\lambda 3727 \AA$, [OIII] $\lambda \lambda 4959,5007 \AA$ superimposed on the cluster spectrum), so that important dust emission is expected in the area. The high dust emission reddening as compared to the stellar content methods (CMD and spectrum) suggests the presence of dust 

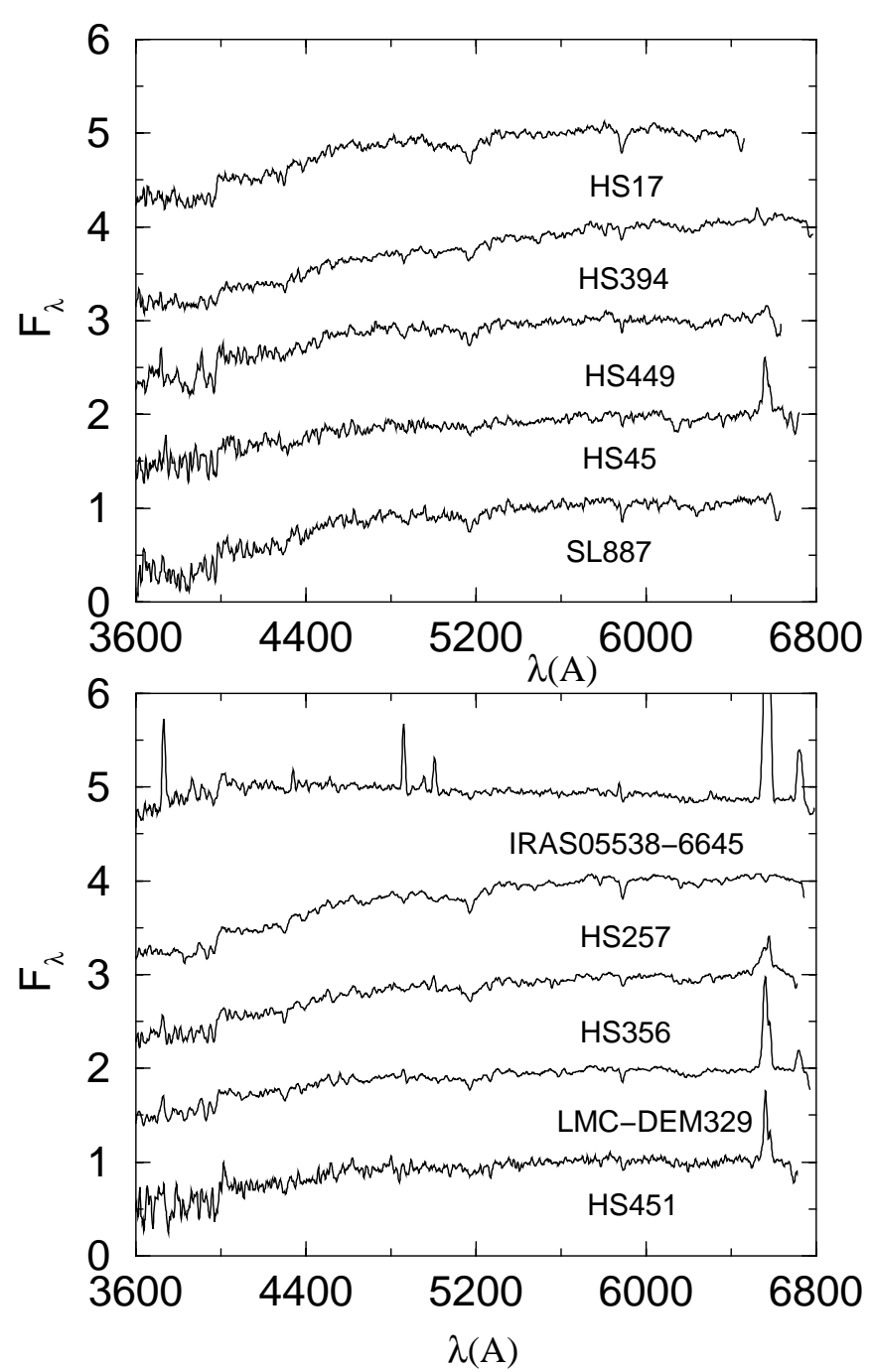

Fig. 4. Same as Fig. 3 for LMC background galaxies

in the cluster background within the SMC, similarly to star cluster directions in the Galaxy (Dutra \& Bica 2000). In contrast to background galaxies, Cloud clusters do not necessarily probe the total internal dust column of the Magellanic Clouds themselves. The age difference for the cluster can be explained by the fact that it is a poorly populated cluster. The turnoff is close to the photometric limit and the field is rich, which can affect significantly the CMD age determination. On the other hand, stochastic effects for bright stars and field contamination for such low mass clusters may cause important uncertainties on the integrated properties (e.g. Geisler et al. 1997). At any rate the bracket $0.5-2 \mathrm{Gyr}$ is a considerable constraint on the cluster age.

OHSC3 (Olszewski et al. 1988) or KMHK 362 (Kontizas et al. 1990) was catalogued as a star cluster. It called our attention owing to the compact nature in ESO/SERC sky survey plates. There is a neighbouring galaxy $\approx 2.3^{\prime}$ to the northeast. This galaxy (New Galaxy 3 ) is an inclined spiral with dimensions $\approx 0.6^{\prime} \times 0.2^{\prime}$ located at $\mathrm{J} 200004^{\mathrm{h}} 57^{\mathrm{m}} 05^{\mathrm{s}}-75^{\circ} 13^{\prime} 08^{\prime \prime}$. We suspected a galaxy pair, but this is not confirmed by the OHSC 3 spectrum. In
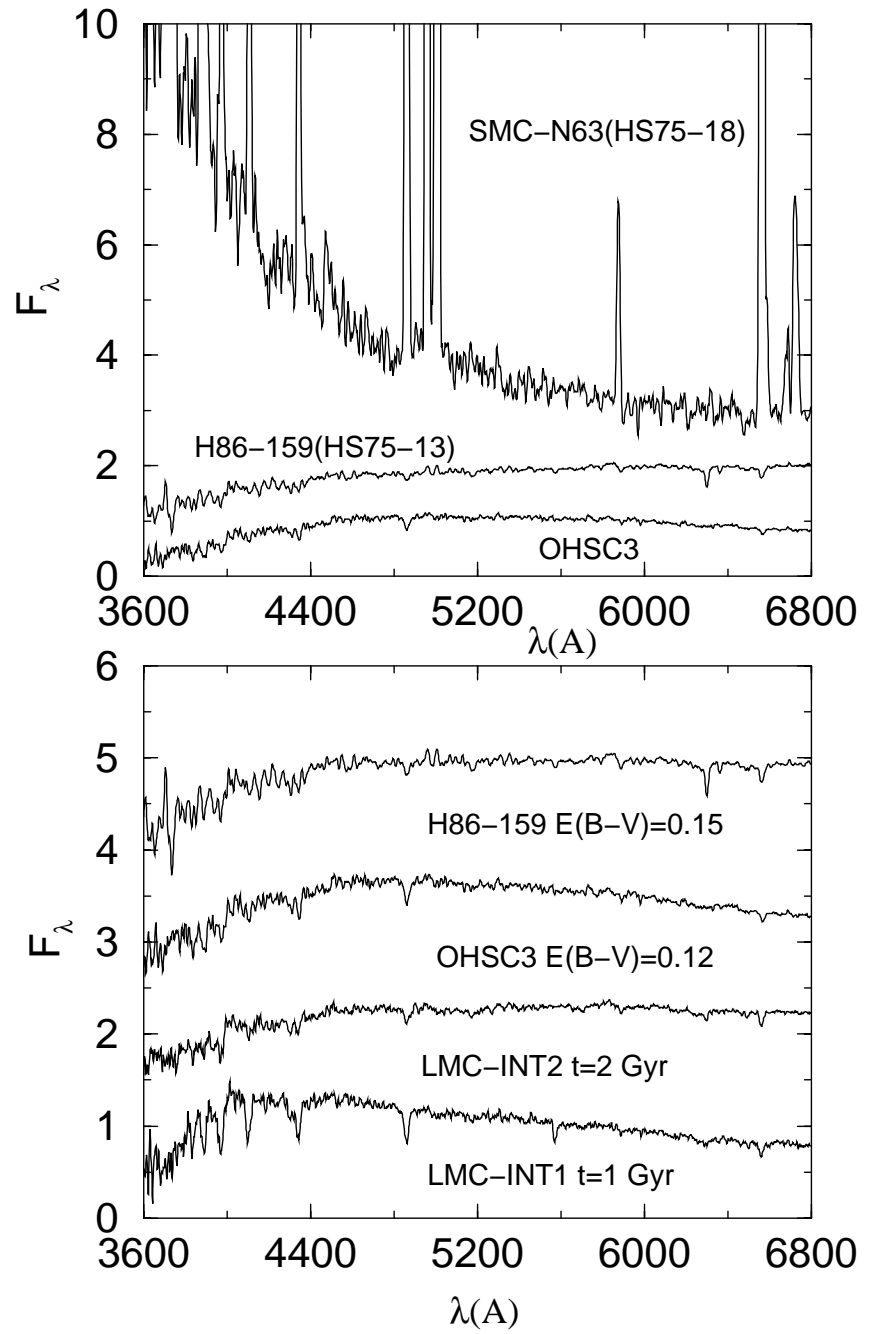

Fig. 5. Upper panel: objects which turned out to be or were confirmed as extended objects belonging to the SMC (H II Region HS 75-18 and star cluster HS 75-13), and LMC (star cluster OHSC3). Lower panel: age and reddening estimates using LMC cluster templates

this direction SFD98 predict $E(B-V)_{\text {FIR }}=0.12$, which was applied in the reddening correction of the OHSC3 spectrum (lower panel of Fig. 5). The spectral properties appear to be intermediate between the two template spectra leading to an age $\approx 1.5$ Gyr.

\section{Reddening-free galaxy templates}

Red stellar population galaxy nuclei are ideal as reddening probes since the spectral distribution is essentially insensitive to age variations of the components, and present a small dependence on metallicity (B88). Blue stellar populations have stronger continuum variations with age distribution of the components.

B88 studied the stellar populations of early and late type galaxies by means of their nuclear integrated spectra. Early and late type galaxy nuclei were studied separately considering also luminosity differences (B88 and references therein). Spectra with similar equivalent widths 
and dereddened continuum distribution were grouped into high signal-to-noise templates. These templates represent the most frequent types of stellar populations found in normal galaxy nuclei. The early type templates E1 to E3 represent a decreasing metallicity sequence among giant early type galaxy nuclei dominated by old (red) stellar populations. E7 represents nuclei dominated by old populations but with significant contribution of 0.5 to $1 \mathrm{Gyr}$ components. S1 to S3 represent a similar metallicity sequence to E1-E3 for red stellar populations occurring in giant spiral galaxy nuclei. Finally, S4 to S7 is a sequence of giant spiral galaxy nuclei with increasing contributions of young stellar populations.

For the spectral comparisons between sample galaxies and templates we employ equivalent widths $(W)$ of strong absorption features. We use as metal features K CaII, CN, $G$ Band, MgI and NaI, together with four Balmer lines. In Table 2 are shown $W$ values for templates and individual galaxies measured with continuum tracings and feature windows following Bica \& Alloin (1986) and Bica et al. (1994). For bluer stellar populations we increased the resolution of spectral properties by creating the intermediate templates S5/S6 $(0.5 \times \mathrm{S} 5+0.5 \times \mathrm{S} 6), \mathrm{S} 6 / \mathrm{S} 7 \mathrm{~A}(0.5 \times \mathrm{S} 6$ $+0.5 \times \mathrm{S} 7)$ and $\mathrm{S} 6 / \mathrm{S} 7 \mathrm{~B}(0.25 \times \mathrm{S} 6+0.75 \times \mathrm{S} 7)$. This procedure in turn provides a higher resolution in reddening determinations since the continuum varies strongly for increasing contents of younger populations in galaxy nuclei. Typical $W$ errors are $\approx 5 \%$ and depend mostly on signalto-noise ratio and uncertainties in the continuum positioning.

Red stellar populations are the most frequent types in the background samples (Figs. 3 and 4 ). In addition to the high reddening accuracy that they can provide (Sect. 6), it is important also to minimize possible observational uncertainties by creating templates from galaxies observed in the same observing runs. For these purposes we built new red stellar population templates using South Polar Cap galaxies (Sect. 2.2) and some galaxies in common with B88, which have $E(B-V)_{\mathrm{FIR}}<0.02$ (Table 3) to avoid dust cirrus (Sect. 2.2). Measurements of $W$ s for these galaxies (Table 2) allowed us to classify them into types E1, E2 or E3. We dereddened the spectra using $E(B-V)_{\mathrm{FIR}}$ values and Seaton's (1979) galactic extinction law. At this stage, the E2 and E3 member galaxies turned out to be very similar in terms of continuum distribution. Therefore we adopted two reference spectra T1 and T23 as counterparts in the present study of the E1 and E2/E3 templates. Members of T1 and T23 are indicated in Table 3. The remaining galaxies with somewhat higher reddening values (Table 3 ) have either internal reddening as dusty ellipticals (Ferrari et al. 1999) and/or foreground contribution. It is possible to determine a spectroscopic reddening value $E(B-V)$ by fitting the observed galaxy spectrum to that of the corresponding template with similar $W$ s and applying continuum corrections according to Seaton's law. We provide in Col. 4 of Table 3 results for individual galaxies in the templates $\mathrm{T} 1$ and $\mathrm{T} 23$ themselves and additional red stellar population galaxies in the same regions.

Table 3 indicates that dust emission and spectroscopic reddening values are consistent for small amounts of reddening. The average of spectroscopic reddening values in the present South Polar Cap sample (13 galaxies) is $E(B-V)=0.02 \pm 0.01$, which is consistent with SFD98's estimate (Sect. 2.2).

\section{Reddening towards the Magellanic Clouds}

For each background galaxy we searched for the template spectrum with comparable $W_{\mathrm{S}}$ (Table 2) which was assumed as the reddening-free reference stellar population. The resulting templates are shown in Col. 2 of Table 4 . Making use of Seaton's law and varying the reddening amount we dereddened the observed galaxy spectrum to match the template continuum distribution. The upper panel of Fig. 6 illustrates the reddening determination for a red stellar population galaxy (HS 394). Note the important reddening effect in the observed spectrum. The lower panel illustrates a blue stellar population nucleus (IRAS 05338-6645). The reddening values obtained by this spectroscopic procedure are given in Col. 3 of Table 4 . For comparison purposes the dust emission reddening $E(B-V)_{\mathrm{FIR}}$ value is shown in Col. 4 . Finally, in Col. 5 we give the HI column density in units of $10^{19}$ atoms $\mathrm{cm}^{-2}$ (Mathewson \& Ford 1984).

The uncertainties in the matching of the continuum distributions is small $\epsilon E(B-V) \approx 0.01$. The largest source of uncertainties arises from the determination of the stellar population. For red stellar populations this uncertainty is typically $\epsilon E(B-V) \approx 0.02$, and somewhat larger for blue stellar populations $(\epsilon E(B-V) \approx 0.05)$.

Out of $36 \mathrm{SMC}$ and LMC background galaxies, 31 show good agreement $(\delta E(B-V) \leq 0.10)$ between the spectroscopic and dust emission reddening values (Table 4 ), leading to a rms of differences of 0.04. One case of significant difference (HS 75-8) is behind the SMC main body, and the remaining ones (New Galaxy 1, HS 356, HS 394 and LMC-DEM329) behind the LMC main body. In the latter 5 cases $E(B-V)_{\mathrm{FIR}}$ is larger and H I column densities are important (Table 4 ). Since the slit apertures are typically $4^{\prime \prime} \times 8-10^{\prime \prime}$ (Sect. 3) and SFD98's pixel dimensions $142^{\prime \prime} \times 142^{\prime \prime}$, a possible explanation is that the dust distribution is patchy with a scale significant smaller than SFD98's pixel. We note that out of the five galaxies with significant differences three have red stellar populations (Table 4) and the spectroscopic reddening values are accurate.

The largest difference occurs for New Galaxy 1 which has a very high reddening value $E(B-V)_{\mathrm{FIR}}=0.68$ (Table 4). This galaxy is projected at the edge of the shell emission nebula LMC-DEM76 (Davies et al. 1976). An alternative explanation to dust small scale variations is that dust in this region may be heated beyond the temperature correction range employed by SFD98. Evidence of a 
Table 2. $W$ s for strong absorption features in the template and individual galaxy spectra

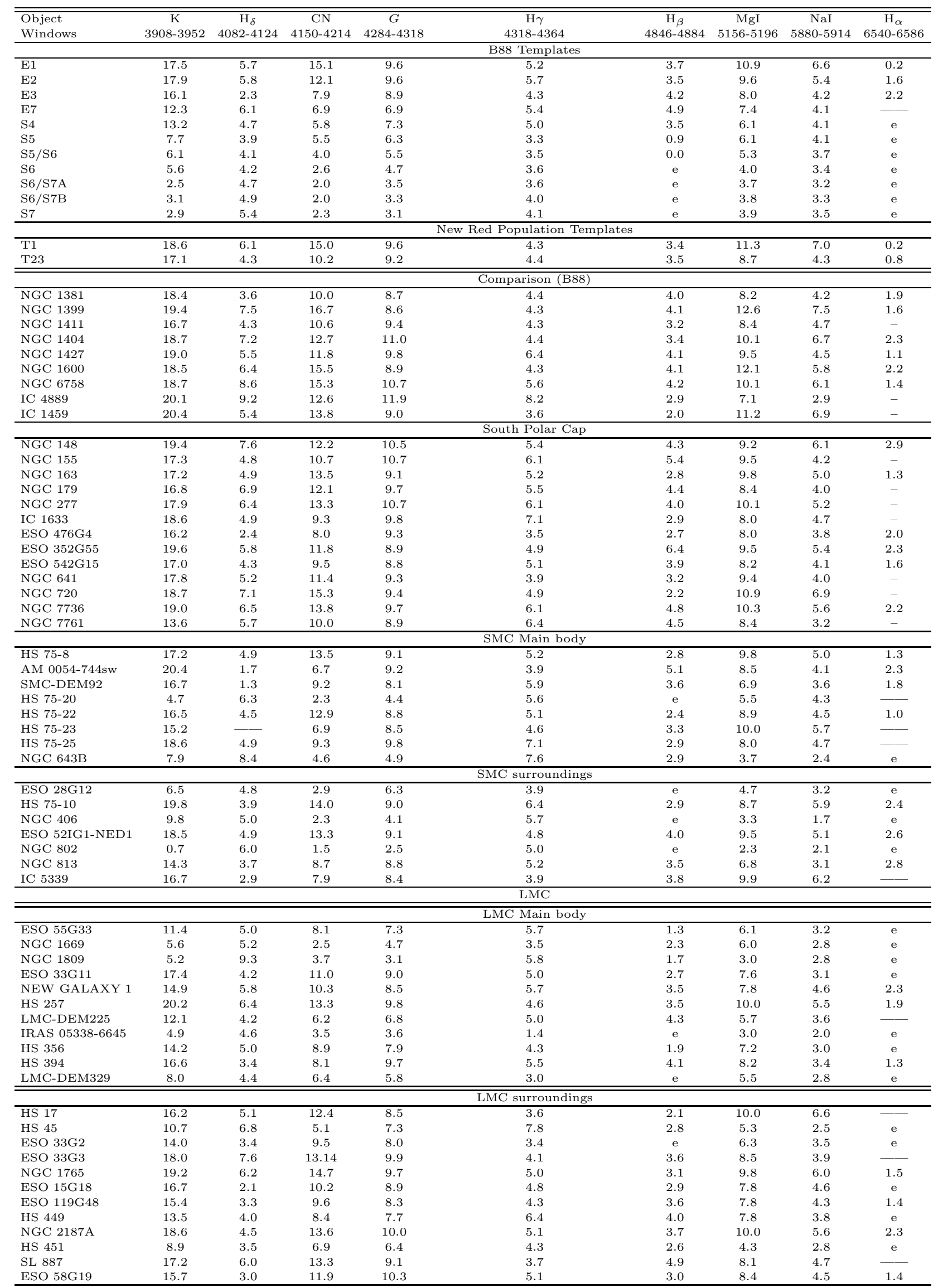

Note: e - indicates Balmer line in emission. 
Table 3. Spectroscopic and dust emission reddening values for observed galaxies in the B88 and South Polar Cap samples

\begin{tabular}{|c|c|c|c|c|}
\hline & & Comparison galaxies & from B88 & \\
\hline Object & Template & comments & $E(B-V)$ & $E(B-V)_{\mathrm{FIR}}$ \\
\hline NGC 1381 & $\mathrm{~T} 23$ & member & 0.01 & 0.01 \\
\hline NGC 1399 & $\mathrm{~T} 1$ & member & 0.01 & 0.01 \\
\hline NGC 1411 & $\mathrm{~T} 23$ & member & 0.01 & 0.01 \\
\hline NGC 1404 & $\mathrm{~T} 1$ & member & 0.02 & 0.01 \\
\hline NGC 1427 & $\mathrm{~T} 23$ & member & 0.01 & 0.01 \\
\hline NGC 1600 & $\mathrm{~T} 1$ & dusty & 0.02 & 0.04 \\
\hline NGC 6758 & $\mathrm{~T} 1$ & dusty & 0.05 & 0.07 \\
\hline IC 4889 & $\mathrm{~T} 23$ & dusty & 0.05 & 0.05 \\
\hline IC 1459 & $\mathrm{~T} 1$ & member & 0.03 & 0.02 \\
\hline \multicolumn{5}{|c|}{ South Polar Cap } \\
\hline Object & Template & Comments & $E(B-V)$ & $E(B-V)_{\mathrm{FIR}}$ \\
\hline NGC 148 & $\mathrm{~T} 23$ & member & 0.02 & 0.02 \\
\hline NGC 155 & $\mathrm{~T} 23$ & & 0.02 & 0.03 \\
\hline NGC 163 & $\mathrm{~T} 1$ & & 0.01 & 0.03 \\
\hline NGC 179 & $\mathrm{~T} 23$ & member & 0.02 & 0.02 \\
\hline NGC 277 & $\mathrm{~T} 1$ & & 0.01 & 0.04 \\
\hline IC 1633 & $\mathrm{~T} 23$ & member & 0.02 & 0.01 \\
\hline $\mathrm{ESO} 476 \mathrm{G} 4$ & $\mathrm{~T} 23$ & member & 0.01 & 0.01 \\
\hline ESO 352G55 & $\mathrm{T} 23$ & & 0.05 & 0.03 \\
\hline ESO $542 \mathrm{G} 15$ & $\mathrm{~T} 23$ & member & 0.01 & 0.02 \\
\hline NGC 641 & $\mathrm{~T} 23$ & member & 0.02 & 0.02 \\
\hline NGC 720 & $\mathrm{~T} 1$ & member & 0.01 & 0.02 \\
\hline NGC 7736 & $\mathrm{~T} 1$ & & 0.03 & 0.03 \\
\hline NGC 7761 & $\mathrm{~T} 23$ & & 0.03 & 0.03 \\
\hline
\end{tabular}

similar effect was pointed out by Dutra \& Bica (2000) for the Galactic Center direction.

The galaxy LMC-DEM225 (Fig. 3) has a rare stellar population type dominated by a red stellar population, but with an important Balmer-line in absorption component in the range $3700 \AA$ to $3900 \AA$. The most similar template in terms of $W$ s available in B88 is E7 (Table 2). The enhanced Balmer lines in the violet region might be explained by a somewhat younger burst (100-500 Myr) in LMC-DEM225, rather than the 1 Gyr burst in E7 (B88). Owing to the stellar population difference we have not determined reddening for this galaxy.

The galaxies projected on SMC surroundings (H I column density $<5010^{19}$ atoms $\mathrm{cm}^{-2}$ ) have an average spectroscopic reddening value of $E(B-V)=0.01 \pm 0.02$. This value is comparable to that of the South Polar Cap galaxies (Sect. 5), showing that at least in the direction of the SMC no significant difference occurs between the Polar region and $b \approx-45^{\circ}$. This value is also comparable to the average dust emission reddening in the SMC surroundings $E(B-V)_{\mathrm{FIR}}=0.03 \pm 0.01$ (Table 4$)$ and that derived by SFD98 in the same region (Sect. 1). Considering the galaxies behind the SMC main body the average spectroscopic reddening is $E(B-V)=0.05 \pm 0.05$ and that from dust emission in the corresponding lines of sight is $E(B-V)_{\mathrm{FIR}}=0.07 \pm 0.04$. By subtracting the average of reddening values in the main body directions from those in the surroundings, both the spectroscopic and dust emission methods predict an internal SMC reddening $\Delta E(B-V)=0.04$ which is consistent with the fact that important H I column density differences occur between these regions (Table 4).

In the LMC surroundings (HI density column $<5010^{19}$ atoms cm $\left.{ }^{-2}\right)$ the average spectroscopic reddening is $E(B-V)=0.06 \pm 0.03$ which is similar to the average dust emission reddening value in the corresponding directions $E(B-V)_{\mathrm{FIR}}=0.08 \pm 0.04$ (Table 4). These values are in turn similar to those derived by SFD98 (Sect. 1). We conclude that in the LMC direction at $b \approx-33^{\circ}$ the Milky Way reddening becomes evident as compared to the Polar Cap. For the LMC main body the average spectroscopic reddening is $E(B-V)=0.12 \pm 0.10$. Considering only the red population galaxies, which provide the most accurate determinations, the result is basically the same $E(B-V)=0.15 \pm 0.11$. This suggests an intrinsic dispersion among lines of sight. We estimate $\Delta E(B-V)=0.06$ between LMC main body directions and surroundings.

\section{Concluding remarks}

Spectra of nuclear regions of galaxies towards the Magellanic Clouds are useful reddening probes in the Milky Way and the Clouds themselves. By means of radial velocities we established the nature of $\approx 20$ objects in terms of background galaxies or star clusters and H II 
Table 4. Spectral and dust emission reddening values for galaxies towards the Magellanic Clouds

\begin{tabular}{|c|c|c|c|c|}
\hline \multicolumn{5}{|c|}{$\mathrm{SMC}$} \\
\hline Object & Template & $E(B-V)$ & $E(B-V)_{\mathrm{FIR}}$ & HI column \\
\hline \multicolumn{5}{|c|}{ Main body } \\
\hline HS 75-8 & $\mathrm{T} 1$ & 0.03 & 0.15 & 675 \\
\hline AM 0054-744sw & $\mathrm{T} 23$ & 0.00 & 0.05 & 136 \\
\hline SMC-DEM92 & $\mathrm{T} 23$ & 0.00 & 0.05 & 130 \\
\hline HS $75-20$ & S6/S7B & 0.10 & 0.05 & 210 \\
\hline HS $75-22$ & $\mathrm{~T} 23$ & 0.04 & 0.04 & 140 \\
\hline HS $75-23$ & $\mathrm{~T} 23$ & 0.00 & 0.04 & 123 \\
\hline HS $75-25$ & $\mathrm{~T} 23$ & 0.10 & 0.10 & 484 \\
\hline NGC 643B & S6 & 0.12 & 0.05 & 82 \\
\hline \multicolumn{5}{|c|}{ surroundings } \\
\hline ESO $28 \mathrm{G} 12$ & S6 & 0.00 & 0.04 & 37 \\
\hline HS $75-10$ & $\mathrm{~T} 1$ & 0.01 & 0.02 & 27 \\
\hline NGC 406 & $\mathrm{~S} 6 / \mathrm{S} 7 \mathrm{~A}$ & 0.00 & 0.02 & 18 \\
\hline ESO 52IG1-NED1 & $\mathrm{T} 23$ & 0.04 & 0.03 & 20 \\
\hline NGC 802 & S7 & 0.00 & 0.02 & 28 \\
\hline IC 5339 & $\mathrm{~T} 23$ & 0.01 & 0.04 & outside \\
\hline \multicolumn{5}{|c|}{ LMC } \\
\hline Object & Template & $E(B-V)$ & $E(B-V)_{\mathrm{FIR}}$ & HI column \\
\hline \multicolumn{5}{|c|}{ Main body } \\
\hline ESO 55G33 & S5 & 0.10 & 0.15 & 50 \\
\hline NGC 1669 & S6 & 0.10 & 0.05 & 75 \\
\hline NGC 1809 & S7 & 0.23 & 0.24 & 75 \\
\hline ESO 33G11 & $\mathrm{T} 23$ & 0.09 & 0.13 & 55 \\
\hline NEW GALAXY 1 & $\mathrm{~T} 23$ & 0.04 & 0.68 & 136 \\
\hline HS 257 & $\mathrm{~T} 23$ & 0.15 & 0.24 & 141 \\
\hline IRAS 05538-6645 & $\mathrm{S} 7$ & 0.20 & 0.13 & 87 \\
\hline HS 356 & S4 & 0.00 & 0.13 & 158 \\
\hline HS 394 & $\mathrm{~T} 23$ & 0.32 & 0.50 & 446 \\
\hline LMC-DEM329 & $\mathrm{S} 56$ & 0.02 & 0.22 & 186 \\
\hline \multicolumn{5}{|c|}{ surroundings } \\
\hline HS 17 & $\mathrm{~T} 23$ & 0.06 & 0.06 & 40 \\
\hline HS 45 & S5 & 0.02 & 0.12 & 44 \\
\hline ESO 33G2 & S4 & 0.08 & 0.13 & outside \\
\hline ESO 33G3 & $\mathrm{T} 1$ & 0.09 & 0.11 & outside \\
\hline NGC 1765 & $\mathrm{~T} 1$ & 0.03 & 0.03 & 7 \\
\hline ESO $15 \mathrm{G} 18$ & $\mathrm{~T} 23$ & 0.10 & 0.10 & outside \\
\hline ESO 119G48 & $\mathrm{T} 23$ & 0.01 & 0.02 & outside \\
\hline HS 449 & $\mathrm{~T} 23$ & 0.01 & 0.06 & 25 \\
\hline NGC 2187A & $\mathrm{T} 1$ & 0.07 & 0.10 & 27 \\
\hline HS 451 & S6 & 0.05 & 0.07 & 5 \\
\hline SL 887 & $\mathrm{~T} 23$ & 0.07 & 0.08 & outside \\
\hline ESO 58G19 & $\mathrm{T} 23$ & 0.10 & 0.11 & outside \\
\hline
\end{tabular}

regions belonging to the Clouds. The radial velocities of the observed galaxies behind the Magellanic Clouds reveal an interesting spatial distribution: most of the brighter galaxies in the main body of the LMC are in the range $4000<V\left(\mathrm{kms}^{-1}\right)<6000$, while for the SMC they are more distant in the range $10000<V\left(\mathrm{~km} \mathrm{~s}^{-1}\right)<20000$. 

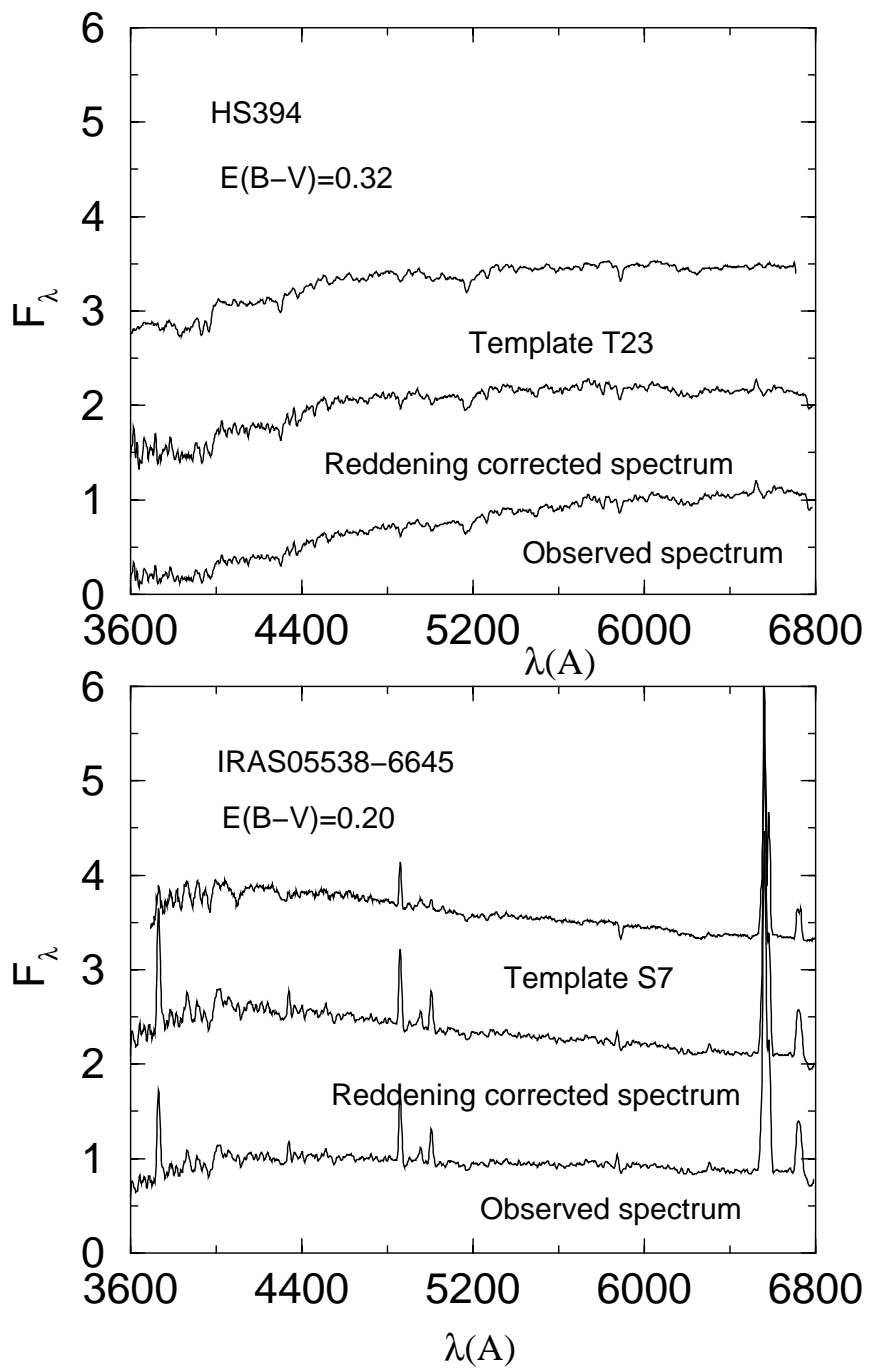

Fig. 6. Upper panel: example of reddening determination for a red stellar population nucleus. Lower panel: example of reddening determination for a blue stellar population nucleus

We estimated reddening values by comparing observed galaxy spectra with reddening-free templates of similar stellar populations. We inferred the reddening distribution throughtout the Clouds by means of 18 galaxies in the main bodies and 18 in the surroundings. The SMC foreground reddening $\left(E(B-V)_{\mathrm{MW}}=0.01\right)$ is comparable to that of the South Polar Cap, while that of the LMC, at a lower galactic latitude is higher $\left(E(B-V)_{\mathrm{MW}}=0.06\right)$. Both Clouds are quite transparent, at least in the sampled lines of sight. The derived average internal reddening values are $E(B-V)_{\mathrm{i}}=0.04$ and 0.06 , respectively for the SMC and LMC. The resulting statistics on reddening values are likely to be skewed toward lower reddening values, since heavily reddened galaxies are less likely to be detected, especially in the most crowded central regions in the Clouds.

Following the reddening study in the Milky Way by Dutra \& Bica (2000), which analyses reddening values derived from dust emission and stellar content of star clusters, the present method probes the foreground and in- ternal reddening in the Clouds by means of background galaxies. We conclude that the dust emission and stellar population reddening values towards the Clouds agree well for $86 \%$ of the present sample. The significant differences found are in the sense of larger $E(B-V)_{\mathrm{FIR}}$ values. Possible explanations are patchy dust distribution in a scale smaller than SFD98's resolution or dust heated above the range of the temperature map.

In order to map out in detail the dust distribution in the Magellanic Clouds large samples of background galaxies are necessary. We note that several of the present main body galaxies are not obvious on Sky Survey images, because of crowding and proximity to extended objects in the Clouds. A fundamental question is whether more reddened zones exist in the Clouds, especially in the LMC. It would be important to carry out higher resolution imaging to identify galaxies for spectroscopy in large telescopes.

Acknowledgements. We thank the CASLEO staff for hospitality and support during the observing runs. The authors acknowledge use of the CCD and data acquisition system supported under U.S. National Science Foundation grant AST-90-15827 to R. M. Rich. We have made use of the LEDA database, (http://leda.univ-lyon1.fr), and the NASA/IPAC Extragalactic Database (NED) which is operated by the Jet Propulsion Laboratory, California Institute of Technology, under contract with the National Aeronautics and Space Administration. We use images from the Digitized Sky Survey (produced at the Space Telescope Science Institute under U.S. Government grant NAG W-2166) by means of the Canadian Astronomy Data Centre (CADC) interface. This work was partially supported by the Brazilian institutions CNPq and FINEP, the Argentine institutions CONICET, ANPCyT and SECYT (UNC), and the VITAE and Antorchas foundations.

\section{References}

Ahumada, A. V., Clariá, J. J., Bica, E., \& Piatti, A. E. 2000, A\&AS, 141, 79

Arp, H. C., \& Madore, B. F. 1987, in A Catalogue of Southern Peculiar Galaxies and Associations (Cambridge Univ. Press)

Baldwin, J. A., \& Stone, R. P. S. 1984, MNRAS, 206, 241

Bica, E. 1988, A\&A, 195, 76

Bica, E., \& Alloin, D. 1986, A\&A, 162, 21

Bica, E., Alloin, D., \& Schmitt, H. 1994, A\&A, 283, 805

Bica, E., \& Schmitt, H. R. 1995, ApJS, 54, 33

Bica, E., Schmitt, H. R., Dutra, C. M., \& Luz Oliveira, H. 1999, AJ, 117, 238

Bica, E., \& Dutra, C. M. 2000, AJ, 119, 1214

Burstein, D., \& Heiles, C. 1978, ApJ, 225, 40

Burstein, D., \& Heiles, C. 1982, AJ, 87, 1165

Crampton, D., Gussie, G., Cowley, A. P., \& Schmidtke, P. C. 1997, AJ, 114, 2353

Davies, R. D., Elliot, K. H., \& Meaburn, J. 1976, MMRAS, 81, 89

de Oliveira, M., Dutra, C. M., Bica, E., \& Dottori, H. 2000, A\&AS, 146, 57

de Vaucouleurs, G., de Vaucouleurs, A., \& Corwin, H. G. 1976, in Second Reference Catalogue of Bright Galaxies (University of Texas Press) (RC2) 
Dutra, C. M., Bica, E., Clariá, J. J., \& Piatti, A. E. 1998, in The formation and evolution of galaxies, Bol. Acad. de Ciencias de Córdoba, 62, 127

Dutra, C. M., Bica, E., Clariá, J. J., \& Piatti, A. E. 1999, MNRAS, 305, 373

Dutra, C. M., \& Bica, E. 2000, A\&A, 359, 347

Ferrari, F., Pastoriza, M. G., Macchetto, F., \& Caon, N. 1999, A\&AS, 136, 269

Geisler, D., Bica, E., Dottori, H., et al. 1997, AJ, 114, 1920

Gurwell, M., \& Hodge, P. 1990, PASP, 102, 849

Haberl, F., \& Pietsch, W. 1999, A\&AS, 139, 277

Haberl, F., Filipovic, M. D., Pietsch, W., \& Kahabka, P. 2000, A\&AS, 142, 41

Henize, K. G. 1956, ApJS, 2, 315

Hodge, P. W., \& Sexton, J. A. 1966, AJ, 71, 363

Hodge, P. W. 1974, ApJ, 192, 21

Hodge, P. W., \& Wright, F. W. 1974, AJ, 79, 858

Hodge, P. W., \& Snow, T. P. 1975, AJ, 80, 9

Kontizas, M., Morgan, D. H., Hatzidimitriou, D., \& Kontizas, E. 1990, A\&AS, 84, 527

Lauberts, A. 1982, The ESO/Uppsala Surgvey of the ESO (B) Atlas, European Southern Observatory (Garching bei München)

Lindsay, E. M. 1961, AJ, 66, 169

MacGillivray, H. T. 1975, MNRAS, 170, 241
Mathewson, D. S., \& Ford, V. L. 1984, IAU Symp., 108, 125

Meyssonnier, N., \& Azzopardi, M. 1993, A\&AS, 102, 451

Oestreicher, M. O., et al. 1995, A\&AS, 112, 495

Oestreicher, M. O., \& Schmidt-Kaler, Th. 1996, A\&AS, 117, 303

Olszewski, E. W., Harris, H. C., Schommer, R. A., \& Canterna, R. W. 1988, AJ, 95, 84

Osterbrock, D. E. 1989, in Astrophysics of Gaseous Nebulae (Univ. Science Books, Mill Valley, California)

Piatti, A. E., Bica, E., \& Clariá, J. J. 1998, A\&AS, 127, 423

Pietrzyński, G., Udalski, A., Szymański, M., et al. 1998, Acta Astron., 48, 175

Pietrzyński, G., \& Udalski, A. 1999, Acta Astron., 49, 157

Reach, W. T., Wall, W. F., \& Odegard, N. 1998, ApJ, 507, 507

Sandage, A. 1973, ApJ, 183, 711

Schlegel, D. J., Finkbeiner, D. P., \& Davis, M. 1998, ApJ, 500, 525

Seaton, M. J. 1979, MNRAS, 187, 73p

Sérsic, J. L. 1974, ApSS, 28, 365

Shapley, H., \& Lindsay, E. M. 1963, Irish AJ, 6, 74

Udalski, A., Szymański, M., Kubiak, M., et al. 1998, Acta Astron., 47, 319

Wesselink, A. J. 1961, MNRAS, 122, 503

Westerlund, B. E. 1990, A\&AR, 2, 29 